\title{
Online interventions for problem gamblers with and without co-occurring problem drinking: study protocol of a randomized controlled trial
}

\author{
John A. Cunningham ${ }^{1,2,3^{*}}$, David C. Hodgins ${ }^{4}$, Matthew Keough ${ }^{5}$, Christian S. Hendershot ${ }^{1,2}$, Kylie Bennett ${ }^{6}$,
} Anthony Bennett ${ }^{6}$ and Alexandra Godinho ${ }^{1,7}$

\begin{abstract}
Background: The current randomized controlled trial seeks to evaluate whether providing access to an Internet intervention for problem drinking in addition to an Internet intervention for problem gambling is beneficial for participants with gambling problems who do or do not have co-occurring problem drinking.

Methods: Potential participants will be recruited online via a comprehensive advertisement strategy, if they meet the criteria for problem gambling. As part of the baseline measures, problem drinking will also be assessed. Eligible participants ( $N=280$ ) who agree to partake in the study and to be followed up for 6 months will be randomized into one of two versions of an Internet intervention for gamblers: an intervention that targets only gambling issues ( $G$-only) and one that combines a gambling intervention with an intervention for problem drinking $(G+A)$. For problem gamblers who exhibit co-occurring problem drinking, it is predicted that participants who are provided access to the $\mathrm{G}+\mathrm{A}$ intervention will demonstrate a significantly greater level of reduction in gambling outcomes at 6 months compared to those provided access to the G-only intervention.
\end{abstract}

Discussion: This trial will expand upon the current research on Internet interventions for addictions and inform the development of treatments for those with co-occurring problem drinking and gambling.

Trial Registration: ClinicalTrials.gov, NCT03323606. Registered on 24 October 2017.

Keywords: Clinical trial, Randomized controlled trial, Brief interventions, Gambling disorders, Problem gambling, Comorbidity, Problem drinking, Internet intervention, Trial protocol

\section{Background}

A challenge for addressing problem gambling, as with many addictions, is that the majority of people with gambling concerns never seek treatment [1-3]. To address these unmet needs, self-directed treatments have been developed, with the initial efforts focusing on bibliotherapy, and more recently, on Internet interventions. While there is a fairly strong evidence base for bibliotherapy [4-7], the evidence on Internet interventions is still emerging [8-10].

\footnotetext{
* Correspondence: john.cunningham@camh.ca

${ }^{1}$ Centre for Addiction and Mental Health, 33 Russell St., Toronto, ON M5S 2S1, Canada

${ }^{2}$ Department of Psychiatry, University of Toronto, Toronto, Canada Full list of author information is available at the end of the article
}

Parallel to the development of self-help interventions for problem gamblers has been the growing recognition that people experiencing gambling problems often experience other, co-occurring mental health concerns [11-16], including problem drinking [13, 14, 17-20]. An important issue to address in delivering self-help interventions (and, indeed, the delivery of face-to-face therapy) is whether there is an advantage in simultaneously providing interventions for mental health concerns alongside the provision of a gambling intervention, in particular among people who are specifically seeking help for their gambling concerns.

This protocol outlines the second of two randomized controlled trials (RCTs) addressing this question. The

(c) The Author(s). 2018 Open Access This article is distributed under the terms of the Creative Commons Attribution 4.0 International License (http://creativecommons.org/licenses/by/4.0/), which permits unrestricted use, distribution, and 
first trial examined the benefits of combining a gambling Internet intervention with an already validated online intervention for mood and anxiety [9]. The current trial uses a similar approach to test the benefits of combining the same online gambling intervention with a brief intervention for problem drinkers.

\section{Major research questions}

The proposed trial will compare the efficacy of two Internet interventions: an intervention that just targets gambling issues (G-only) versus one that contains an intervention for problem drinking in addition to an intervention for gamblers $(G+A)$. Outcome variables for gambling will measure the reduction in gambling severity and frequency of gambling. The outcome for a reduction in alcohol use will be measured by the reduction in the frequency of drinking during the past week. The primary hypotheses are:

Hypothesis 1: For problem gamblers with cooccurring problem drinking, it is predicted that participants provided access to the $\mathrm{G}+\mathrm{A}$ website will display significantly reduced gambling outcomes at the 3 - and 6-month follow-ups compared to those provided access to the G-only website. For problem gamblers without co-occurring problem drinking, there will be no significant difference between those provided access to the G-only and G + A websites at the 3- and 6-month follow-ups.

Hypothesis 2: For problem drinkers, it is predicted that participants provided access to the $\mathrm{G}+\mathrm{A}$ website will display significantly reduced drinking outcomes at the 3- and 6-month follow-ups compared to those provided access to the G-only website. For participants who are not problem drinkers, there will be no significant difference in drinking between those provided access to the G-only and G + A websites at the 3- and 6-month follow-ups.

Hypothesis 3: Problem gamblers with co-occurring problem drinking who receive the $\mathrm{G}+\mathrm{A}$ intervention and reduce the amount they drink between the baseline and 3-month follow-ups will display significantly improved gambling outcomes at the 6-month follow-up compared to problem drinkers who receive the $\mathrm{G}+\mathrm{A}$ intervention but experience no decrease in their drinking.

Follow-ups at 3 and 6 months were chosen as it is unknown whether the intervention will have the predicted impact, and we wished to optimize the possibility of finding an effect by employing a short-term follow-up.

\section{Methods/design}

\section{Participants}

Participants will be recruited using a comprehensive online and in-print advertisement strategy targeting Manitoba residents (e.g., local newspaper ads, social media ads on Facebook, and website ads on Yahoo and Google AdWords restricted to Manitoba residents). Advertisements will target gamblers who self-identify as being concerned about their gambling and are interested in participating in a study that aims to "find ways to help people who are worried about their gambling." Prospective participants, 18 years or older who report gambling in the past 30 days, will be directed to complete a brief web-based screener that assesses problem gambling severity. The eligibility of individuals will be determined by their willingness to be followed up, and a current score of 3 or more on the Problem Gambling Severity Index (PGSI) [21]. Prior treatment access will be measured; however, it will not be used to determine eligibility as the intent of this trial is to evaluate the impact of the interventions within a real-world community setting. Similarly, cooccurring depression, anxiety, and illicit drug consumption will be measured but will not be used as exclusion criteria. Finally, while recruitment is not specifically targeted to attract problem drinking, it is anticipated that the trial will contain participants with and without co-occurring problem drinking. Based on the frequency of co-occurring problem drinking and gambling in the general population, it is estimated that approximately $50 \%$ of the sample will have co-occurring problem drinking [14, 22]. Participants in the first trial (examining the impact of combining an Internet intervention for gambling with one for mood and anxiety) will not be eligible to participate in the current trial.

\section{Study design and procedures}

The proposed study is a two-arm double-blinded parallelgroup RCT comparing two online interventions over a 6month period: an intervention targeting gambling issues only versus one that contains interventions for hazardous alcohol consumption. Potential participants, who respond to the advertisement by clicking on the link or visiting the website, will be provided first with a brief description of the study prior to completing an eligibility screener. Individuals who are found to be eligible will be asked to provide contact information in the form of an email address. Subsequently, potential participants will be sent an email with a link to an online consent form. Participants will be asked to provide their telephone number and mailing address as additional contact information, as well as to provide permission for study staff to contact them via phone or mail for follow-up surveys if correspondence by email is unsuccessful. Those who complete the consent form and provide a real postal address will be sent a link to complete the baseline questionnaire and will be randomized into one of the two experimental conditions. Randomization will be stratified on age, sex, and prior use of treatment using an automatized algorithm to ensure a random distribution of prognostic indicators at baseline. To reduce attrition in the study, those who complete the 
baseline questionnaire and access the online intervention will receive a \$10 Amazon.ca gift certificate. At 3 and 6 months post-randomization, all participants will receive an email request to complete a follow-up survey in the form of a hyper-linked web address. In addition, up to two email reminders for follow-up survey completion will be sent to participants to promote retention in the study. Furthermore, participants who complete the 3-month follow-up will be sent a $\$ 20$ gift certificate and those who complete the 6-month follow-up will be sent an additional $\$ 30$ gift (i.e., a total honorarium of $\$ 60$ for each participant including the amount given at the baseline). Lastly, participants in both the G-only and the $\mathrm{G}+\mathrm{A}$ interventions will receive an additional three emails encouraging the use of each respective intervention. See Fig. 1 for a Consort diagram summarizing the study design.

\section{Ethical approval}

This study, including methods and design, has been approved by the standing ethics review committee of the Centre for Addiction and Mental Health (CAMH).

\section{Interventions}

\section{G-only}

The gambling only Internet intervention will consist of selfchange tools developed by Hodgins et al. [23] that have been adapted to an online interface. Three separate trials have demonstrated the significant impact of these tools on gambling $[4,6,7]$, and their successful use within an online format has also been previously documented $[8,9]$. The intent of these tools is to provide individuals with both behavioral and cognitive strategies that assist in the recovery from or a reduction in gambling.

\section{$G+A$}

The gambling plus alcohol intervention will consist of the G-only intervention as well as access to a study specific version of Check Your Drinking (CYD). The CYD contains a brief 18 -item screener that has been designed to assess the quantity and frequency of drinking, and the severity of hazardous drinking [24]. Following the completion of the 18-item screener, the user is provided with a personalized report that contains normative feedback (i.e., it compares the person's drinking with the drinking of others in the general population of the same age, sex, and country of origin, such as Canada, the U.S.A., and the U. K.). The CYD has been subjected to five randomized controlled trials from two independent research groups, all of which displayed its significant impact by reducing hazardous alcohol consumption compared to controls [24-28].

Both versions of the website contain a link to a page listing resources to contact in case of a crisis.

\section{Measures}

Assessment points and instruments are presented in Fig. 2, and the SPIRIT checklist is available as Additional File 1.

\section{Baseline survey}

The baseline survey will assess demographic characteristics (e.g., age, sex, education, marital status, income, and

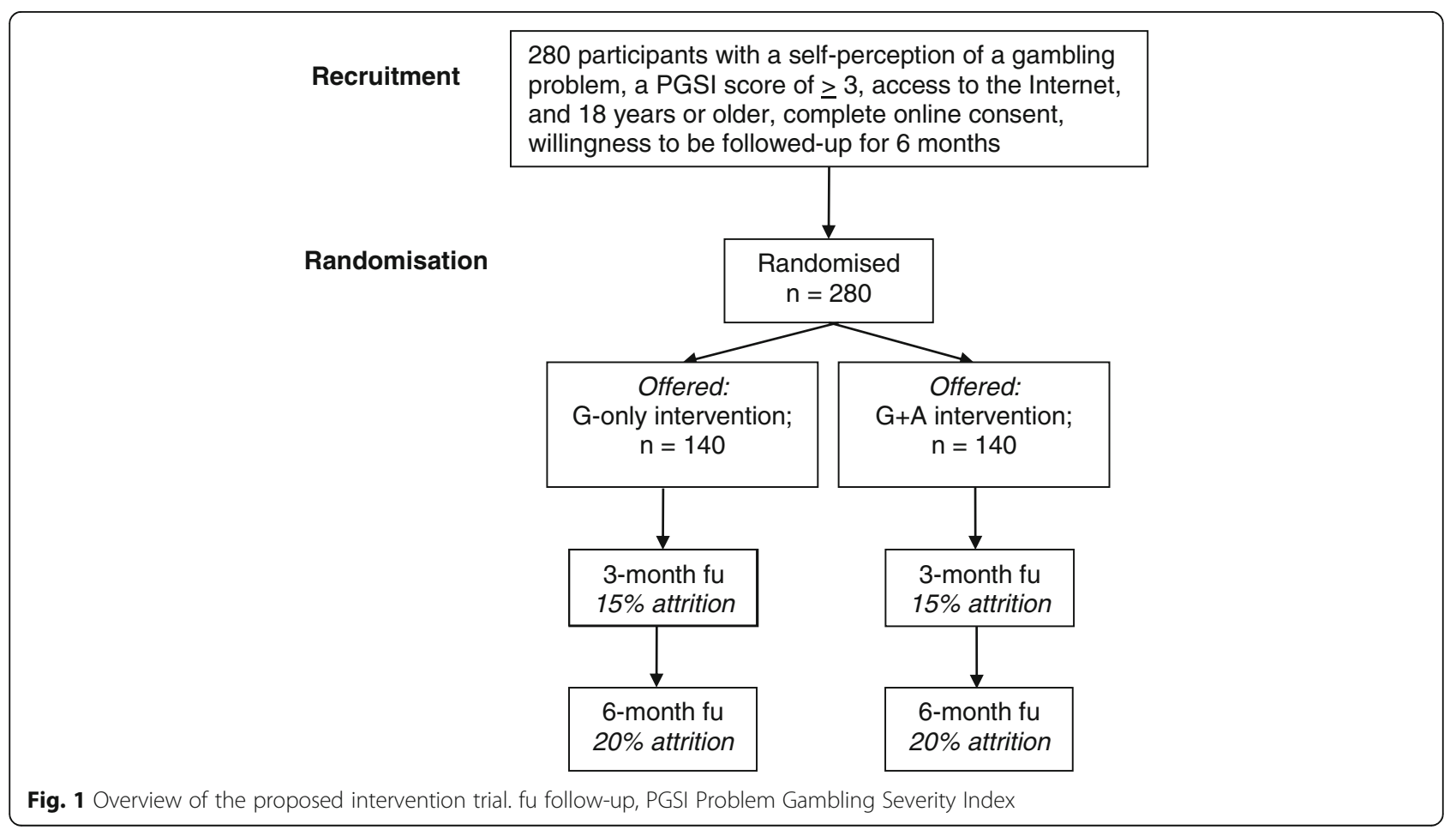




\begin{tabular}{|c|c|c|c|c|}
\hline & \multicolumn{4}{|c|}{ STUDY PERIOD } \\
\hline & Enrolment & Allocation & Post-allocation & Close-out \\
\hline TIMEPOINT & $-t_{1}$ & 0 & $t_{3 m}$ & $t_{6 m}$ \\
\hline \multicolumn{5}{|l|}{ ENROLMENT: } \\
\hline \multirow{2}{*}{$\begin{array}{l}\text { Eligibility screen } \\
\text { Informed consent }\end{array}$} & $\mathrm{X}$ & & & \\
\hline & $\mathrm{X}$ & & & \\
\hline Allocation & & $\mathrm{X}$ & & \\
\hline \multicolumn{5}{|l|}{ INTERVENTIONS: } \\
\hline \multirow[t]{2}{*}{ [G-only] } & & & $\mathrm{X}$ & \\
\hline & & & $\mathrm{X}$ & \\
\hline \multicolumn{5}{|l|}{ ASSESSMENTS: } \\
\hline \multicolumn{5}{|l|}{ Baseline Variables } \\
\hline \multirow{2}{*}{$\begin{array}{l}\text { Demographics } \\
\text { Gambling frequency } \\
\text { and severity }\end{array}$} & $\mathrm{X}$ & & & \\
\hline & $\mathrm{X}$ & & & \\
\hline Treatment history & $\mathrm{X}$ & & & \\
\hline Treatment goals & $\mathrm{X}$ & & & \\
\hline \multicolumn{5}{|l|}{ Primary Outcome } \\
\hline \multirow{2}{*}{$\begin{array}{l}\text { Problem gambling } \\
\text { severity } \\
\text { \# days gambled per } \\
\text { month }\end{array}$} & $\mathrm{X}$ & & $\mathrm{X}$ & $\mathrm{X}$ \\
\hline & $\mathrm{X}$ & & $\mathrm{X}$ & $\mathrm{X}$ \\
\hline \multicolumn{5}{|l|}{ Secondary Outcomes } \\
\hline \multirow{2}{*}{$\begin{array}{c}\text { \# drinks per typical } \\
\text { week }\end{array}$} & $\mathrm{X}$ & & $\mathrm{X}$ & $\mathrm{X}$ \\
\hline & & & & \\
\hline \multirow{2}{*}{$\begin{array}{l}\text { Other gambling- } \\
\text { related measures } \\
\text { Hazardous alcohol } \\
\text { consumption }\end{array}$} & $\mathrm{X}$ & & $\mathrm{X}$ & $\mathrm{X}$ \\
\hline & $\mathrm{X}$ & & $\mathrm{X}$ & $\mathrm{X}$ \\
\hline Mental Health Issues & $\mathrm{X}$ & & $\mathrm{X}$ & $\mathrm{X}$ \\
\hline Illicit drug use & $\mathrm{X}$ & & $\mathrm{X}$ & $\mathrm{X}$ \\
\hline Quality of life & $X$ & & $\mathrm{X}$ & $\mathrm{X}$ \\
\hline \multirow[t]{2}{*}{ Ongoing treatment use } & $\mathrm{X}$ & & $\mathrm{X}$ & $\mathrm{X}$ \\
\hline & $X$ & & $\mathrm{X}$ & $\mathrm{X}$ \\
\hline
\end{tabular}

Fig. 2 Study assessment points and instruments

employment status), clinical characteristics (such as gambling frequency and severity, prior treatment use, problem drinking, illicit drug use in the past year, mental health issues, i.e., depressive/anxiety symptoms and general psychological distress), and quality of life (i.e., using the World Health Organization Quality of Life 8-item survey [29]).

The severity of problem gambling will be measured using the past year PGSI and the past 3-month version of the NORC DSM-IV Screen for Gambling Problems (NODS), which indicates DSM-IV defined severity [30, 31]. The NODS has been previously employed within a 1-year follow-up study to assess brief treatment and its utility as an outcome measure [32]. Additionally, the mean number of days gambled in the past 3 months, the amount spent on gambling, and forms of gambled engaged in will be assessed. The use of treatment services for gambling concerns will be measured by asking participants to identify services accessed from a comprehensive list of treatment forms, as used in previous studies $[4,6]$. Lastly, participants 
will also be asked to identify a treatment goal (quit or reduced gambling) and their perceived success in achieving that goal over the following 3 and 6 months on a Likert scale ranging from 0 "not at all" to 10 "extremely." The primary outcome variables for gambling will be the NODS and number of days gambled.

Hazardous alcohol consumption will be measured using a past 3-month version of the Alcohol Use Disorders Identification Test, with the frequency of heavy drinking occasions modified for female and male participants (i.e., five or more drinks for males and four or more drinks for females) [33]. A score of 8 or more will indicate problem drinking. The primary outcome variable for alcohol consumption will be number of drinks consumed in a typical week.

The severity of depressive and anxiety symptoms will be measured using the Patient Health Questionnaire 9item scale (PHQ-9) [34] and the Generalized Anxiety Disorder 7-item scale (GAD-7) [35], respectively. In addition, general psychological distress will be measured using the Kessler 10 questionnaire (K10), as this measure has been found to be responsive to change over time. The $\mathrm{K} 10$ has been well validated and is appropriate for self-administration, as it is brief, simple, and easy to comprehend [36, 37].

\section{Follow-up surveys}

The 3- and 6-month surveys will employ the same measures as those assessed at baseline using a past 3-month timeframe.

\section{Use of interventions}

A count of participant logins into the intervention, as well as the various web pages accessed within the Gonly intervention (as well as use of the CYD), will be available. These data will be used to investigate whether participants' degree of involvement with the intervention is associated with their success at recovering from gambling or reducing gambling severity. The degree of G-only intervention use by participants will be operationalized according to the previously recommended methods [38, 39]. The number of times a participant accesses the site, the number of tools the participant uses (as assessed by page views, form completions, etc.), and the length of involvement with the site (e.g., use of the site over time) will be recorded.

\section{Power analysis}

Efforts will be made to recruit a total sample of 280 participants, and we anticipate that 224 participants will be successfully followed up at 6 months (i.e., estimated 20\% attrition based on previous research of online interventions, albeit targeting hazardous alcohol use) [40]. This sample size will provide sufficient power to conduct the proposed statistical analyses necessary to test the aforementioned hypotheses. Based upon previous data collected by Hodgins et al. $[4,6]$ on gambling frequency and NODS scores, and assuming that a correlation of 0.5 exists between baseline and follow-up measures, then power $=.0 .80$ and $\alpha=.05$. This sample size is sufficient to detect a difference of at least two gambling days per month between both experimental conditions in both the 3- and 6-month follow-up periods. Smaller differences in gambling days per month may not be clinically meaningful. Similarly, the proposed sample size will also be sufficiently powered to detect a 1point difference of NODS scores at the 6-month follow-up. Calculations are based upon a repeated measures ANOVA model (G.Power 3.1.9).

\section{Data analysis}

The two primary hypotheses of this study, which focus on comparing outcomes between groups for gambling and drinking respectively, will be analyzed using linear mixed-effect models with random intercepts. This will allow us to use all available participant data within the models, employing a restricted maximum likelihood to handle missing data. Separate analyses will be conducted for each primary outcome variable (i.e., NODS score, mean number of day gambled in the past month, and, for drinking, the number of drinks consumed in a typical week). For secondary analyses, mixed-effects models will be conducted to examine the effect of moderators on primary variables, that is, additional fixed-effect interactions will be included in exploratory models (e.g., extent of intervention use, participants' sex, severity of gambling and drinking at baseline, and co-occurrence of mental health concerns). In addition, secondary analyses will also examine predictors of treatment use, as well as the effect of accessing treatment on primary outcomes. The third hypothesis, comparing the potential moderating effect of reductions in drinking on gambling severity, will be examined using Hayes's Process macro. This method uses a state-of-the-art regression-based conditional process approach [41] to estimate covariances, variances, and means. Missing data will be handled using a maximum likelihood approach. Finally, we will examine whether changes in quality of life are related to improvements in gambling outcomes.

\section{Discussion}

Many problem gamblers are also problem drinkers $[13,14$, 17-20], with a lifetime prevalence as measured by nationally representative samples ranging from $45 \%$ to $73 \%$ [14, 22]. Heavy drinking often occurs while problem gamblers are engaging in gambling activities [42], resulting in increased risky gambling behavior [43-45]. Further, co-occurring problem drinking negatively impacts the treatment outcomes of problem gamblers (see the systematic 
review by Merkouris et al. [46]). Targeting problem drinking by problem gamblers may have the dual benefits of reducing both the problem drinking itself and of acting as a mediator for reductions in problem gambling behavior.

The proposed study seeks to determine whether providing simultaneous Internet interventions for gambling and drinking is of benefit for those with these cooccurring problems. More specifically, it will evaluate whether there is an incremental benefit to providing access to a problem drinking Internet intervention $(\mathrm{G}+$ A intervention) in addition to an Internet intervention for problem gambling (G-only intervention) for individuals with gambling problems who do or do not have co-occurring problem drinking. A limitation of the current trial is its short follow-up period (6-month follow-up). If the trial finds that the addition of a brief alcohol intervention to an online gambling intervention is beneficial at this time point, then future research examining any sustained impact of the intervention would be merited. The results of this study will enhance our understanding of Internet interventions for addictions in general, as well as inform how treatments can be developed and matched to the care of and needs for those with co-occurring problem gambling and drinking.

\section{Trial Status}

Protocol version: 1.

Date recruitment began: 20 November 2017.

Approximate date recruitment will be completed: December 2018.

\section{Additional file}

Additional file 1: SPIRIT 2013 Checklist: Recommended items to address in a clinical trial protocol and related documents. (DOC $121 \mathrm{~kb}$ )

\section{Abbreviations}

CAMH: Centre for Addiction and Mental Health; CYD: Check Your Drinking; DSM-4: Diagnostic Statistical Manual Version 5; G + A: G-only intervention plus access to Check Your Drinking, an Internet intervention for hazardous drinking; GAD-7: Generalized Anxiety Disorder 7-item scale; K10: Kessler Psychological Distress Scale; NODS: NORC Diagnostic Screen for Gambling Problems; PGSI: Problem Gambling Severity Index; PHQ-9: Patient Health Questionnaire 9-item scale; RCT: Randomized controlled trial

\section{Acknowledgements \\ This research was undertaken in part thanks to funding from the Canada Research Chairs program in support of JAC (the Canada Research Chair in Addictions) and CSH (the Canada Research Chair in Etiology and Treatment of Alcohol Use Disorders). Support to CAMH for salary and infrastructure has been provided by the Ministry of Health and Long-Term Care. The views expressed in this article do not necessarily reflect those of the Ministry of Health and Long-Term Care.}

\section{Funding}

This research is funded by the Manitoba Gambling Research Program of Manitoba Liquor and Lotteries; however, the findings and conclusions of this paper are solely those of the authors and do not necessarily represent the views of Manitoba Liquor and Lotteries. The funding body has no role or influence on the design of the study, on the collection, analysis, and interpretation of data, or on writing the manuscript.

\section{Availability of data and materials}

There are no applicable data since this is a protocol paper. The study materials are available from the corresponding author.

\section{Authors' contributions}

All authors have made an intellectual contribution to this research trial. JAC is the principal investigator of the trial, with overall responsibility for the project. JAC, DCH, MK, and CSH conceived the design and wrote the grant application. AG and JAC developed the protocol and wrote the first draft of this manuscript. $A B$ and $K B$ developed the online tool, and conceived of and developed the online RCT platform for conducting the current study. All authors have contributed to the manuscript drafting process and have read and approved the final manuscript.

\section{Ethics approval and consent to participate}

This research was approved by the Ethics Review Board of CAMH. All participants will provide informed consent as part of the study registration process

\section{Competing interests}

The authors declare that they have no competing interests. DCH has received consulting fees from the Australian National University to develop the online gambling tool. $K B$ and $A B$ are owners and employees of eHub Health Pty Ltd., which has a commercial license to deliver the interventions.

\section{Publisher's Note}

Springer Nature remains neutral with regard to jurisdictional claims in published maps and institutional affiliations.

\section{Author details}

${ }^{1}$ Centre for Addiction and Mental Health, 33 Russell St., Toronto, ON M5S 2S1, Canada. ${ }^{2}$ Department of Psychiatry, University of Toronto, Toronto, Canada. ${ }^{3}$ Austalian National University, Canberra, Australia. ${ }^{4}$ Department of Psychology, University of Calgary, Calgary, Canada. ${ }^{5}$ Department of Psychology, University of Manitoba, Winnipeg, Canada. ${ }^{6}$ eHub Health Pty Ltd, Collector, Australia. 'Dalla Lana School of Public Health, University of Toronto, Toronto, Canada.

Received: 3 February 2018 Accepted: 4 May 2018

Published online: 25 May 2018

References

1. Cunningham JA. Little use of treatment among problem gamblers. Psychiatr Serv. 2005;56(8):1024-5.

2. Suurvali H, Hodgins DC, Toneatto T, Cunningham JA. Treatment-seeking among Ontario problem gamblers: results of a population survey. Psychiatr Serv. 2008:59:1343-6.

3. Slutske WS. Natural recovery and treatment-seeking in pathological gambling: results of two U.S. national surveys. Am J Psychiatry. 2006; 163(2):297-302.

4. Hodgins DC, Currie SR, el-Guebaly N. Motivational enhancement and self-help treatments for problem gambling. J Consult Clin Psychol. 2001; 69(1):50-7.

5. Hodgins DC, Currie S, el-Guebaly N, Peden N. Brief motivational treatment for problem gambling: a 24-month follow-up. Psychol Addict Behav. 2004; 18(3):293-6.

6. Hodgins DC, Currie SR, Currie G, Fick GH. Randomized trial of brief motivational treatments for pathological gamblers: More is not necessarily better. J Consult Clin Psychol. 2009;77(5):950-60.

7. Abbott M, Bellringer M, Vandal A, Hodgins DC, Palmer D, Preez $L$, Sullivan $\mathrm{S}$, Feigin $\mathrm{V}$. Effectiveness of problem gambling brief telephone interventions: A randomized controlled trial. In: Auckland University of Technology, prepared for the New Zealand Ministry of Health; 2012.

8. Hodgins DC, Fick GH, Murray R, Cunningham JA. Internet-based interventions for disordered gamblers: study protocol for a randomized controlled trial of online self-directed cognitive-behavioural motivational therapy. BMC Public Health. 2013;13:10. 
9. Cunningham JA, Hodgins DC, Bennett K, Bennett A, Talevski M, Mackenzie CS, Hendershot CS. Online interventions for problem gamblers with and without co-occurring mental health symptoms: Protocol for a randomized controlled trial. BMC Public Health. 2016;16:624.

10. Merkouris SS, Rodda SN, Austin D, Lubman DI, Harvey P, Battersby M, Cunningham J, Lavis T, Smith D, Dowling NA. GAMBLINGLESS: FOR LIFE study protocol: a pragmatic randomised trial of an online cognitive-behavioural programme for disordered gambling. BMJ Open. 2017;7(2):e014226.

11. Bischof A, Meyer C, Bischof G, Kastirke N, John U, Rumpf HJ. Comorbid Axis I-disorders among subjects with pathological, problem, or at-risk gambling recruited from the general population in Germany: results of the PAGE study. Psychiatry Res. 2013;210(3):1065-70.

12. Desai RA, Potenza MN. Gender differences in the associations between pastyear gambling problems and psychiatric disorders. Soc Psychiatry Psychiatr Epidemiol. 2008;43(3):173-83.

13. Kessler RC, Hwang I, LaBrie R, Petukhova M, Sampson NA, Winters KC, Shaffer HJ. DSM-IV pathological gambling in the National Comorbidity Survey Replication. Psychol Med. 2008;38(9):1351-60.

14. Petry NM, Stinson FS, Grant BF. Comorbidity of DSM-IV pathological gambling and psychiatric disorders: Results from the National Epidemiologic Survey on Alcohol and Related Conditions. J Clin Psychol. 2005:66(5):564-74.

15. Lorains FK, Cowlishaw S, Thomas SA. Prevalence of comorbid disorders in problem and pathological gambling: systematic review and meta-analysis of population surveys. Addiction. 2011;106(3):490-8.

16. Martin RJ, Usdan S, Cremeens J, Vail-Smith K. Disordered gambling and comorbidity of psychiatric disorders among college students: An examination of problem drinking, anxiety and depression. J Gambl Stud. 2014;30(2):321-33.

17. Barnes GM, Welte JW, Tidwell MC, Hoffman JH. Gambling and Substance Use: Co-occurrence among Adults in a Recent General Population Study in the United States. Int Gambl Stud. 2015;15(1):55-71.

18. el-Guebaly N, Patten SB, Currie S, Williams JV, Beck CA, Maxwell CJ, Wang JL. Epidemiological associations between gambling behavior, substance use \& mood and anxiety disorders. J Gambl Stud. 2006;22(3):275-87.

19. Martins SS, Ghandour LA, Lee GP, Storr CL. Sociodemographic and substance use correlates of gambling behavior in the Canadian general population. J Addict Dis. 2010;29(3):338-51.

20. Welte JW, Barnes GM, Tidwell MC, Hoffman JH, Wieczorek WF. Gambling and Problem Gambling in the United States: Changes Between 1999 and 2013. J Gambl Stud. 2015;31(3):695-715.

21. Ferris J, Wynne H. The Canadian Problem Gambling Index: Final Report. In: Canadian Centre on Substance Abuse; 2001.

22. Cunningham-Williams RM, Cottler LB, Compton WM 3rd, Spitznagel EL. Taking chances: problem gamblers and mental health disorders-results from the St. Louis Epidemiologic Catchment Area Study. Am J Public Health. 1998;88(7):1093-6.

23. Hodgins DC, Makarchuk K. Becoming a winner. Defeating problem gambling. In: Edmonton: AADAC; 2002.

24. Cunningham JA, Wild TC, Cordingley J, van Mierlo T, Humphreys K. A randomized controlled trial of an internet-based intervention for alcohol abusers. Addiction. 2009;104(12):2023-32.

25. Doumas DM, Hannah E. Preventing high-risk drinking in youth in the workplace: A web-based normative feedback program. J Subst Abus Treat. 2008;34(3):263-71.

26. Doumas DM, Haustveit T. Reducing heavy drinking in intercollegiate athletes: Evaluation of a web-based personalized feedback program. The Sport Psychologist. 2008;22:213-29.

27. Doumas DM, McKinley LL, Book P. Evaluation of two Web-based alcohol interventions for mandated college students. J Subst Abus Treat. 2009;36(1):65-74.

28. Cunningham JA, Murphy M, Hendershot CS. Treatment dismantling pilot study to identify the active ingredients in personalized feedback interventions for hazardous alcohol use: Randomized controlled trial. Addict Sci Clin Pract. 2014;e9:22

29. Schmidt S, Muhlan H, Power M. The EUROHIS-QOL 8-item index: psychometric results of a cross-cultural field study. Eur J Pub Health. 2006; 16(4):420-8.

30. Toce-Gerstein M, Volberg RA. The NODS-CLiP: A new brief screen for pathological gambling. Paper presented at the International Symposium on Problem Gambling and Co-Occurring Disorders. Mystic, CT; 2004.
31. Wulfert E, Hartley J, Lee M, Wang N, Franco C, Sodano R. Gambling screens: Does shortening the time frame affect their psychometric properties? J Gambl Stud. 2005;21(4):521-36.

32. Hodgins DC. Using the NORC DSM Screen for Gambling Problems as an outcome measure for pathological gambling: psychometric evaluation. Addict Behav. 2004;29(8):1685-90.

33. Saunders JB, Aasland OG, Babor TF, De La Fuente JR, Grant M. Development of the Alcohol Use Disorders Identification Test (AUDIT): WHO collaborative project on early detection of persons with harmful alcohol consumptionII. Addiction. 1993;88:791-804.

34. Kroenke K, Spitzer RL, Williams JBW. The PHQ-9 - Validity of a brief depression severity measure. J Gen Intern Med. 2001;16(9):606-13.

35. Spitzer RL, Kroenke K, Williams JBW, Lowe B. A brief measure for assessing generalized anxiety disorder - The GAD-7. Arch Intern Med. 2006:166(10):1092-7.

36. Kessler RC, Andrews G, Colpe LJ, Hiripi E, Mroczek DK, Normand SLT, Walters EE, Zaslavsky AM. Short screening scales to monitor population prevalences and trends in non-specific psychological distress. Psychol Med. 2002;32(6):959-76.

37. Brooks RT, Beard J, Steel Z. Factor structure and interpretation of the K10. Psychol Assessment. 2006;18(1):62-70.

38. Danaher BG, Smolkowski K, Seeley JR, Severson HH. Mediators of a successful web-based smokeless tobacco cessation program. Addiction. 2008;103(10):1706-12.

39. Strecher VJ, McClure J, Alexander G, Chakraborty B, Nair V, Konkel J, Greene S, Couper M, Carlier C, Wiese C, et al. The role of engagement in a tailored web-based smoking cessation program: randomized controlled trial. J Med Internet Res. 2008;10(5):e36.

40. Cunningham JA, Shorter GW, Murphy M, Kushnir V, Rehm J, Hendershot CS. Randomized Controlled Trial of a Brief Versus Extended Internet Intervention for Problem Drinkers. Int J Behav Med. 2017:5:760-7.

41. Hayes AF. Introduction to Mediation, Moderation, and Conditional Process Analysis: A Regression-Based Approach. New York: Guildford Press; 2013.

42. Baron E, Dickerson M. Alcohol Consumption and Self-Control of Gambling Behaviour. J Gambl Stud. 1999:15(1):3-15.

43. Cronce JM, Corbin WR. Effects of alcohol and initial gambling outcomes on within-session gambling behavior. Exp Clin Psychopharmacol. 2010;18(2): $145-57$.

44. Giacopassi D, Stitt BG, Vandiver M. An Analysis of the Relationship of Alcohol to Casino Gambling Among College Students. J Gambl Stud. 1998; 14(2):135-49.

45. Ellery M, Stewart SH. Alcohol affects video lottery terminal (VLT) gambling behaviors and cognitions differently. Psychol Addict Behav. 2014;28(1):206-16.

46. Merkouris SS, Thomas SA, Browning CJ, Dowling NA. Predictors of outcomes of psychological treatments for disordered gambling: A systematic review. Clin Psychol Rev. 2016:48:7-31.

\section{Ready to submit your research? Choose BMC and benefit from:}

- fast, convenient online submission

- thorough peer review by experienced researchers in your field

- rapid publication on acceptance

- support for research data, including large and complex data types

- gold Open Access which fosters wider collaboration and increased citations

- maximum visibility for your research: over $100 \mathrm{M}$ website views per year

At BMC, research is always in progress.

Learn more biomedcentral.com/submissions 